\title{
The molecular mechanisms of transition between mesenchymal and amoeboid invasiveness in tumor cells
}

\author{
K. Paňková · D. Rösel · M. Novotný • \\ Jan Brábek
}

Received: 1 July 2009/Revised: 10 August 2009/Accepted: 11 August 2009/Published online: 26 August 2009

(C) The Author(s) 2009. This article is published with open access at Springerlink.com

\begin{abstract}
Tumor cells exhibit at least two distinct modes of migration when invading the $3 \mathrm{D}$ environment. A single tumor cell's invasive strategy follows either mesenchymal or amoeboid patterns. Certain cell types can use both modes of invasiveness and undergo transitions between them. This work outlines the signaling pathways involved in mesenchymal and amoeboid types of tumor cell motility and summarizes the molecular mechanisms that are involved in transitions between them. The focus is on the signaling of the Rho family of small GTPases that regulate the cytoskeleton-dependent processes taking place during the cell migration. The multiple interactions among the Rho family of proteins, their regulators and effectors are thought to be the key determinants of the particular type of invasiveness. Mesenchymal and amoeboid invasive strategies display different adhesive and proteolytical interactions with the surrounding matrix and the alterations influencing these interactions can also lead to the transitions.
\end{abstract}

Keywords Tumor cells - Invasiveness - Migration ·

Mesenchymal · Amoeboid · Transition · MAT ·

Rho GTPases

\section{Introduction}

The ability of tumor cells to spread to distant locations in the body and to form metastases is the most life-threatening

K. Paňková · D. Rösel · M. Novotný · J. Brábek ( $ه)$

Department of Cell Biology, Faculty of Science,

Charles University in Prague, Vinicna 7,

12843 Prague 2, Czech Republic

e-mail: brabek@natur.cuni.cz aspect of cancer. Therefore, an understanding of the mechanisms underlying this feature is crucial. Metastasis is a complex process involving several steps: first, tumor cells must break away from the primary tumor and invade locally, through the barriers of the surrounding extracellular matrix (ECM) normally present in tissues. Next, the further spread of tumor cells to secondary tumor sites occurs via the bloodstream or the lymph vessel system; therefore, the metastasizing tumor cells must be capable of intravasation, survival in the bloodstream or lymphatic system, and extravasation. In addition, tumor cells must be able to colonize distant sites (reviewed in [1]).

A local invasion to adjacent tissue is one of the early steps in the metastatic process and one of the key determinants of the metastatic potential of tumor cells. In order to overcome the ECM barriers, tumor cells develop different strategies. The cells can spread either collectively, retaining their intracellular junctions, or individually. The conversion from epithelial cells to motile individually migrating cells is an intensively studied phenomena known as epithelial-mesenchymal transition (EMT; reviewed in [1]). This work concentrates on the single cell migration strategies of tumor cells, referred to as amoeboid and mesenchymal.

The amoeboid and mesenchymal types of invasiveness are two modes of migration that are mutually interchangeable: suppression or enhancement of the activity of specific molecular pathways that determine either of the modes can cause a switch to the other type of invasiveness-this is called mesenchymal-amoeboid transition (MAT) or amoeboid-mesenchymal transition (AMT), respectively. While EMT is a quite rigid process following relatively extensive alterations in gene transcription, MAT/AMT involves rapid changes in a migratory mode that arise as a reply to the current specifics of the environment. 
The transitions may play a role in different stages of the metastatic process when a certain microenvironment requires phenotypical adaptation of the tumor cells. So far, several molecular mechanisms inducing MAT/AMT have been reported (as described below). A study of the mechanisms that may possibly trigger MAT/AMT helps us to understand the plasticity in the migration strategies of tumor cells. This is important for the development of a cancer treatment that would efficiently suppress tumor cell invasiveness through the inhibition of both modes of migration.

\section{An overview of the invasive strategy of individual tumor cells}

Mesenchymal type of invasiveness

The mesenchymal type of tumor cell migration can be compared to fibroblast-like motility. Apart from fibroblasts, keratinocytes, endothelial cells, and some tumor cells also use this mode of migration. Cells with the mesenchymal type of motility have a specific elongated spindle-like shape. In 3D matrices, cells are polarized, creating an obvious leading edge with one or more leading pseudopods and the lagging cell body (containing nucleus, cytoplasm, and organelles) that can be easily distinguished (see Fig. 1).

The translocation of the mesenchymally migrating cells begins with the formation of actin-rich filopodia and lamellipodia at the leading edge. This process is driven by the small GTPases from the Rho family, primarily by Rac and $\mathrm{Cdc} 42$ [2, 3]. Adhesive interactions with the ECM are present on both the cell poles, and the contractile actin stress fibers attached to them generate traction forces between the anterior and posterior cell edge [4]. Clustered integrins give rise to the focal adhesions that then recruit ECM-degrading proteolytical enzymes to perform pericellular ECM remodeling and generate the path for migrating cells $[5,6]$. The velocity of mesenchymal cell migration in
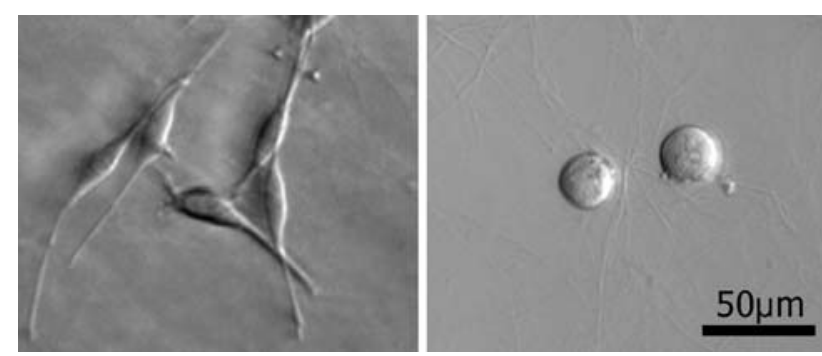

Fig. 1 Different morphologies of the invasive tumor cells. Left mesenchymal morphology of K4 sarcoma cells. Right amoeboid morphology of A3 sarcoma cells (representative modulation contrast image recorded at an invasion depth of $50 \mu \mathrm{m}$ )
3D matrices is approximately $0.1-0.5 \mu \mathrm{m} / \mathrm{min}$ [7]. The rather low speed is caused by the relatively slow turnover of focal adhesions during the translocation [8].

Amoeboid-like invasiveness

The amoeboid migration is named after the specific type of motility of amoeba, which is characterized by cycles of expansion and contraction of the cell body mediated by the cortically localized actin and myosin [9]. Amoeboid-like movement in higher eukaryotes has been described in leukocytes [10,11] and certain types of tumor cells [12-15]. Tumor cells that adopt an amoeboid style of migration have a characteristic rounded shape in 3D substrates. The typical morphology of a tumor cell with amoeboid-like invasiveness in a 3D environment can be seen in Fig. 1.

The enhanced contractility of cells that use amoeboidlike invasive strategies, promoted by the Rho/ROCK signaling pathway $[12,14]$, enables them to squeeze through gaps in the ECM fibers and adapt their bodies to the preexisting spaces $[10,11,13]$, or to exert a sufficient force to deform the surrounding ECM [14-16]. The tension maintained by cortical actomyosin results in membrane blebbing that contributes to the cell motility [17]. Amoeboidly migrating cells move in 3D substrates independently of ECM degradation [13, 14]. The low-adhesion attachment to the substrate enables cells that adopt an amoeboid movement to translocate in the 3D environment at relatively high velocities, ranging from $2 \mu \mathrm{m} / \mathrm{min}$ observed on A375m2 melanoma cells [12] to $25 \mu \mathrm{m} / \mathrm{min}$, which represents the peak migration velocity of lymphocytes in collagen gel [18].

A summary of the distinct characteristics of the mesenchymal and amoeboid type of invasiveness is outlined in Table 1.

Individual tumor cell invasiveness at the molecular level

In general, a single mesenchymal tumor cell migration can be described in three steps: (1) the initial cell polarization and formation of the leading protrusion, which leads to (2) the interaction of the leading edge with ECM. Cell-ECM contacts trigger downstream signaling events that are followed by (3) the contraction of the rear of the cell and displacement of the cell.

Mesenchymal migration begins with the extension of lamellipodia (flat 2D protrusions containing a branched network of the actin filaments) and filopodia (thin rod-like projections composed of the parallel actin fibers) at the cell edge. Rho-family small GTPases Rac and Cdc42 mediate actin polymerization by the regulation of WASP/WAVE proteins $[19,20]$. The interaction of N-WASP and WAVE2 
Table 1 A comparison of the main phenotypic characteristics of the mesenchymal and amoeboid modes of invasiveness

\begin{tabular}{ll}
\hline Mesenchymal & Amoeboid \\
\hline $\begin{array}{l}\text { Morphology } \\
\text { Elongated }\end{array}$ & Rounded \\
$\begin{array}{l}\text { Attachment to the ECM } \\
\text { Via integrin clusters creating focal }\end{array}$ & $\begin{array}{c}\text { Weak, short-term, integrins } \\
\text { diffused in the membrane }\end{array}$ \\
contacts and adhesions & \\
$\begin{array}{l}\text { Migration in the ECM } \\
\text { ECM degradation, remodeling }\end{array}$ & $\begin{array}{c}\text { Proteolysis-independent } \\
\text { pushing through the ECM }\end{array}$ \\
$\begin{array}{l}\text { Organization of actin cytoskeleton } \\
\text { Actin meshwork (leading edge), } \\
\text { stress fibers (traversing the cell) }\end{array}$ & $\begin{array}{l}\text { Contractile actin cortex } \\
\text { Velocity of the locomotion }\end{array}$ \\
$\begin{array}{l}\text { Low } \\
\text { Cell membrane extensions }\end{array}$ & High \\
Filopodia and lamellipodia & Intensive blebbing \\
\hline
\end{tabular}

with Arp2/3 promotes nucleation of actin filaments and formation of the actin network at the leading edge [21]. Cdc42 induces formation of filopodia [3, 22] and affects the initial cell polarity through the regulation of microtubules (MTs) [23].

Lamellipodia and filopodia at the leading edge are stabilized by the interactions of focal contacts with ECM. The key components of the focal contacts are integrins, the transmembrane receptors that bind the common components of ECM and mediate a mechanical linkage between ECM and actin cytoskeleton. The activation and co-clustering of integrins in the focal contacts is mediated by an adaptor protein called talin that couples integrins with actin cytoskeleton [24]. Integrin clusters recruit several adaptor proteins (e.g., paxillin, vinculin, zyxin) and signaling proteins [focal adhesion kinase (FAK), Src] and develop into more stable focal adhesions (reviewed in [25]). Attachment to the ECM is crucial for the mesenchymally migrating cells. Blocking of the $\beta 1$ integrins in mesenchymally invading HT-1080 fibrosarcoma cells has been shown to lead to a loss of motility [13]. The formation of the focal adhesions is associated with the Rho signaling-dependent rearrangement of the actin cytoskeleton to the long parallel actin stress fibers [3]. Clustered integrins recruit extracellular proteolytical enzymes such as matrix metalloproteinases (MMPs) and serine proteases [5, 6] to perform local pericellular remodeling of the ECM. The generation of the tube-like migratory pathways facilitates tumor cell migration.

The actual displacement of a cell depends on the traction force that arises from the contraction of stress fibers [4]. Contractility of the actin cytoskeleton is maintained largely by Rho signaling. Rho in GTP-bound form activates its effector Rho-associated serine-threonine protein kinase (ROCK) that inactivates myosin-light-chain phosphatase (MLCP) [26]. MLCP dephosphorylates myosin II light chain (MLC2). Myosin-light-chain kinase (MLCK) has an antagonistic effect: it phosphorylates MLC2, and ROCK has also been found to directly phosphorylate MLC2 [27]. Once the MLC2 is phosphorylated (P-MLC2), the activity of myosin II ATPase is enhanced, and myosin II becomes engaged in more efficient interactions with actin filaments and thus increases cell contractility (reviewed in [28]).

The other pathway involved in the regulation of cell contractility involves Cdc42 signaling [29]. Cdc42 acts through its downstream effector myotonic dystrophy kinase-related Cdc42-binding kinase (MRCK) [30] and this signaling has an inhibitory effect on MLCP [29]. The ratio of activities of MLC2 phosphorylating and P-MLC2 dephosphorylating regulators determines the level of actomyosin contractility.

Interestingly, Rho/ROCK signaling has been shown to be dispensable for the mesenchymal type of migration of the BE colon carcinoma and SW962 squamous cell carcinoma cells [12]. The activated Cdc42/MRCK pathway sufficiently compensates the loss of contractility in mesenchymally migrating $\mathrm{BE}$ colon carcinoma cells after inhibition of the Rho/ROCK pathway [29]. Only the simultaneous inactivation of both kinases, ROCK and MRCK, results in a significant decrease in the cell motility of the mesenchymal BE colon carcinoma cells [29].

In contrast to the mesenchymal mode of invasion, in the amoeboid-like migration, the contractile cortical actomyosin network is crucial for the generation of the motive force $[28,31]$. The submembranous actin is attached to the membrane by linking proteins from the 4.1 band superfamily-ezrin, radixin, and moesin (reviewed in [32]). Rho/ROCK signaling maintains the contractility of the membrane-attached actomyosin cortex [12, 14], which maintains cell tension. As observed in Amoeba proteus, the intrinsic hydrostatic pressure can lead to onset of a movement [31]. The intracellular pressure results either in the detachment of the actomyosin network from the membrane or in the rupture of the actomyosin cortex and formation of spherical membrane herniations, so-called membrane blebs [17]. The basic difference between the formation of lamellipodia in the mesenchymal motility and membrane blebbing is that the blebs are formed by the inflow of a cytoplasm [17]; thus, the driving force of their formation is not the actin polymerization as it is in lamellipodia [2]. The formation of the membrane blebs predominantly at the leading edge of the migrating cells [17] may be triggered by increased pressure on the membrane in the direction of the movement, which is caused by the increased accumulation of contractile actomyosin at the posterior cortex —as observed in Dictyostelium [9]—or by 
the depletion of cortex-membrane linking proteins at the blebbing cell edge and the local disassembly of actin [17]. Once the bleb is formed, the actomyosin network beneath the membrane is restored [33] and the bleb is either retracted or used for further translocation.

It has been proposed that the extension and stabilization of the bleb is driven by a further inflow of the cytoplasm and interactions with the ECM. The exact nature of the cell-ECM attachments in amoeboid migration is the subject of further research. In Dictyostelium amoebae, there are no integrins expressed and the substrate-binding forces are low [11]. Similarly, in HT-1080 cell lines, after the induced transition to amoeboid migration, the loss of integrin clusters was observed [13]. The independence of the amoeboid cancer cell migration from the $\beta 1$ integrins was also reported in melanoma cells [34]. This, together with the impaired downstream signaling from $\alpha 2 \beta 1$ integrin complexes identified in primarily amoeboid LS174T cells and HT-1080 cell line with induced amoeboid migration [35], implies a reduced requirement for the formation of integrin-mediated cell-ECM contacts in the amoeboid pattern of translocation in general.

The key marker of the amoeboid invasiveness of tumor cells is its independence from ECM proteolytic degradation that has been found in the several primarily amoeboid cell lines $[12,14]$ and mesenchymal cells after a mesenchymal to amoeboid transition [13]. The migrating strategy of amoeboid cell lines in 3D substrates is based on Rho/ ROCK maintained contractility [12-14]. Originally, the proposed model of amoeboid-like migration in 3D lattices was that cells squeeze through the pre-existing spaces in ECM with no remodeling of the surrounding matrix [12, 13]. However, Rho/ROCK maintained contractility has recently been shown not only to maintain high cell deformability but also to exert sufficient force to structurally change the ECM [14-16, 28].

\section{The main molecular mechanisms underlying MAT/AMT}

MAT/AMT transitions are determined

by the actomyosin contractility

As described previously, amoeboid invasiveness largely relies on actomyosin contractility maintained by Rho/ROCK signaling $[12,14]$. The silencing of the Rho/ROCK pathway in amoeboid tumor cells was the first mechanism that was observed to induce transition from amoeboid to mesenchymal invasiveness [12]. The inactivation of either Rho or ROCK in A375m2 melanoma cells with the blebbing amoeboid-like phenotype results in transition to the mesenchymal-like morphology [12]. Among the important regulators of the Rho/ROCK-maintained cortical actomyosin network is 3-phosphoinositide-dependent protein kinase 1 (PDK1) that has a positive effect on ROCK1 (a ROCK isoform) activity at the plasma membrane [36]. PDK1 promotes ROCK1-driven organization of actomyosin by competing with RhoE, a negative regulator of ROCK1 [36]. Depletion of PDK1 disrupts the organization of MLC2 at the cell cortex and results in the elongated mesenchymal phenotype and impaired motility as observed in A $375 \mathrm{~m} 2$ cells [36].

Rho/ROCK signaling not only drives the amoeboid migration but also impairs Rac-induced lamellipodia formation in cells using the amoeboid type of migration by activating GAP of Rac called ARHGAP22, thereby suppressing the mesenchymal strategy of invasiveness [37]. The silencing of ARHGAP22 in amoeboid A375m2 led to the elongated mesenchymal phenotype with decreased levels of P-MLC2 [37].

Cdc42-mediated signaling has also been found to be important in the maintenance of the amoeboid mode of invasiveness in $\mathrm{A} 375 \mathrm{~m} 2$ melanoma cells [38]. It has been shown that inhibition of the Cdc42 regulator, DOCK10, or its downstream effectors, N-WASP and PAK2, leads to amoeboid-mesenchymal transition [38]. Cdc42 also activates MRCK, which cooperates with ROCK on MLC2 phosphorylation, but due to redundancy with ROCK, inactivation of MRCK only has a moderate effect on A $375 \mathrm{~m} 2$ invasion [29]. This data suggests that the critical role of Cdc42 for amoeboid invasiveness is not the MLC2 phosphorylation but the activation of actin polymerization through its effector, namely N-WASP or PAK2. The interactions among the Rho GTPases and their involvement in MAT/AMT are depicted in Fig. 2.

MAT/AMT induced by inhibition of the initial cell polarization

Rac is believed to be the key regulator of the lamellipodia formation in mesenchymally migrating cells [2, 19, 39]. Inactivation of Rac induces a rounded phenotype in A375P cells and increased levels of P-MLC2 in A $375 \mathrm{~m} 2$ cells seeded on top of thick collagen [37]. The same effect has been observed with the inactivation of either NEDD9 or DOCK3, which both mediate the activation of Rac (DOCK3 is a GEF of Rac, NEDD9 is an adaptor protein creating a complex with DOCK3) [37]. Rac1 also influences the assembly of focal adhesions and the subsequent formation of stress fibers [40]. Moreover, Rac suppresses cell contractility through its effector WAVE2 that negatively regulates the phosphorylation of MLC2 and therefore renders the amoeboid mode of motility unfavorable [37]. Thus, Rac signalization can be considered crucial for the mesenchymal migration of tumor cells, although the 


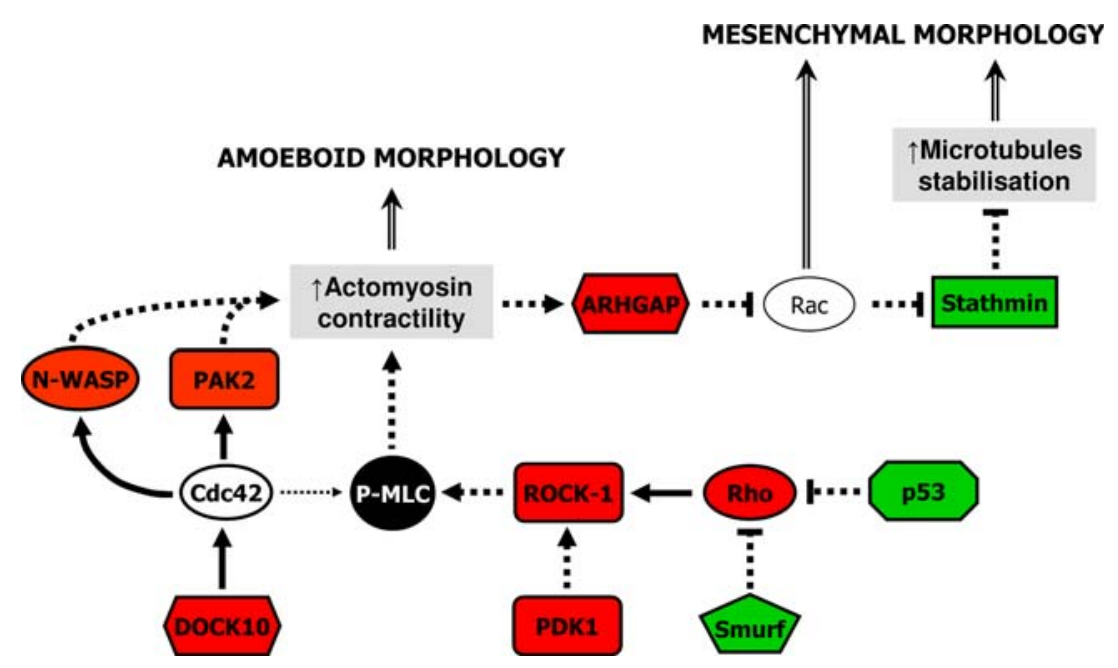

Fig. 2 Interactions among the components of signaling pathways documented to be involved in the MAT/AMT transitions of cells in a 3D environment. The inhibition of the activity of the proteins highlighted in red was shown to trigger amoeboid to mesenchymal transitions. Inactivation of the proteins depicted in green induces a conversion from the mesenchymal to the amoeboid mode of invasiveness. Mesenchymal to amoeboid transitions were also

Rac-induced morphological transitions have not yet been documented within the 3D environment but were observed only on top of the thick collagen [37].

Local inhibition of cell contractility at the leading edge is necessary for the efficient formation of leading protrusions in mesenchymal invasiveness. The depletion of RhoA at the leading edge is maintained by the Smurf1 protein (an E3-ubiquitin ligase) that targets RhoA for the proteolytical degradation [41] and therefore locally impairs Rho/ROCK signaling. Smurf1 is therefore believed to be important for this mode of migration. Consistently, the inhibition of Smurf1 in mesenchymal BE colon carcinoma cells is sufficient to induce transition to amoeboid invasiveness [42].

Cdc42 is involved in multiple pathways crucially regulating cell migration, and therefore the inactivation of Cdc42 leads to the non-motile phenotype [38]. In mouse embryonic fibroblasts, formation of the $\mathrm{Cdc} 42$ induced filopodia is negatively regulated by $\mathrm{p} 53$, a transcription factor that functions as tumor suppressor [43]. The actual mechanisms of this interference are not known, but it is proposed that $\mathrm{p} 53$ acts downstream from $\mathrm{Cdc} 42$ [43]. In mesenchymally migrating A375P melanoma cells in a 3D environment, the loss of $\mathrm{p} 53$ function has been found to: induce transition to the amoeboid phenotype with the rounded morphology; increased RhoA activity, and membrane blebbing [44].

Cdc42 is also the key regulator of cell polarity in fibroblast migration that is maintained by microtubules [23]. The dynamics of MTs in the cell is regulated by several stabilizing and destabilizing proteins that interact either with the tubulin subunits or polymerized MTs. One documented in cells expressing activated Cdc42 (Q61L mutation). Rac, a well-established signaling element essential for mesenchymal movement, is also shown, even though the Rac-dependent morphological transitions were not yet thoroughly documented within the $3 \mathrm{D}$ environment. Solid lines direct connections, dashed lines indirect connections

of the MT-regulating proteins is stathmin (also known as Op18) that contributes to the MT destabilization [45]. It was observed on the HT-1080 fibrosarcoma cells that stathmin 1 (a stathmin isoform) expression enhances migration in 3D matrices, even though it does not influence the invasiveness in a 2D environment [46]. The enhanced migration of the originally mesenchymally invading HT- 1080 cells in 3D assays is coupled with the acquisition of a rounded shape [46] suggesting that stathmin 1 may contribute to MAT. The cell morphology was the only sign of amoeboid invasiveness that Belletti's group observed (in 2008); as yet there is no evidence of stathmin 1 (over)expression-induced changes in the cell surface integrins distribution, increased P-MLC2 levels, or membrane blebbing.

MTs are important for the maintenance of cell polarity in mesenchymally migrating cells [23], but in amoeboidlike migration of neutrophils [10], the role of MTs is opposite. There is evidence that, in neutrophils, a treatment inducing MT disassembly supports the motility of neutrophils by selective activation of ROCK and ROCK-induced increased levels of P-MLC2 [47]. Disruption of MTs appears to be an appropriate signal leading to MAT [46]; however, the role of the destabilization of MTs in amoeboid-like tumor cells has not yet been reported.

MAT/AMT related to proteolytical, adhesive and remodeling interactions with the ECM

The increased proteolysis of ECM by tumor cells expressing extracellular matrix metalloproteinases (MMPs), 
serine proteases, and cathepsins was considered to be the crucial determinant of tumor cell invasiveness until the blocking of the extracellular proteolysis revealed an extracellular proteases-independent mode of invasiveness [13].

Mesenchymally migrating HT-1080 fibrosarcoma cells are able to invade in $3 \mathrm{D}$ collagen lattices after treatment with an inhibitory cocktail in an ECM proteolysis-independent manner, showing the typical features of amoeboid invasiveness such as rounded morphology, integrin diffusion in the plasma membrane, and the use of cortical actomyosin contractility during the migration [13, 14, 35]. The induced amoeboid migration of HT-1080 cells after the inhibition of proteolysis is associated with a decreased cell surface expression of $\alpha 2 \beta 1$ integrins and a reduced level of phosphorylated FAK [35], suggesting a lower requirement for formation and signaling from focal adhesions. Although the amoeboid invasiveness is supposed to be $\beta 1$ integrin-independent [34], suppression of the $\beta 1$ integrin-mediated adhesion to the ECM in mesenchymally migrating cells was not shown to be a sufficient mechanism for the induction of MAT [35].

A recent study has shown how tumor cells are able to use Rho/ROCK-dependent and Rho/ROCK-independent modes of invasiveness with respect to the spatial organization of surrounding collagen fibers [16]. In mesenchymally migrating MDA-MB-231 breast carcinoma cells, Rho/ ROCK independent migration can only be used when the collagen fibers are pre-aligned perpendicularly to the tumorECM boundary, whereas Rho/ROCK mediated contractility is used in this cell type for the active, protease-independent reorganization of filaments in cases where the filaments are not pre-aligned [16].

An overview of MAT/AMT transitions documented in the 3D environment is shown in more detail in Table 2.

Recently, Sabeh et al. [48] suggested that the amoeboid invasiveness of tumor cells observed in vivo can only occur under specific conditions and may not be an effective and widespread alternative to single tumor cell migration [48], as was previously thought [12-14]. The authors suggest that protease-independent mechanisms of cell migration are only plausible when the collagen network is devoid of the covalent cross-links that characterize normal tissues. They emphasize that descriptions of proteinase-independent, amoeboid-like cancer cell behavior are largely drawn from in vitro assays using model 3D ECM constructs that do not reproduce the key structural characteristics displayed by native type I collagen networks in vitro or in vivo. Specifically, ECM constructs in these studies were reconstituted from pepsin-extracted type I collagen, a proteolytic process that removes the nonhelical telopeptides situated at the $\mathrm{N}$ - and $\mathrm{C}$-terminal ends of native collagen molecules. These collagen telopeptides play an important role in fibrillogenesis and contain critical lysine residues, which, after lysyl oxidase-dependent oxidation in vivo, support the intermolecular covalent cross-links necessary for stabilizing gel architecture [48].

The authors compared the invasiveness of HT-1080 and MDA-MB-231 cells in matrices from pepsin-extracted and intact full-length collagen gels and found much more efficient invasion of both cell types in matrices from pepsin-extracted collagens. The authors concluded that MT1-MMP-independent invasion only proceeds when the structural pores formed in collagen gel networks are no longer stabilized by the covalent cross-links that define fibril architecture and structural rigidity. However, neither the HT-1080 nor MDA-MB-231 cells used in this study are cells that primarily use the amoeboid mode of invasiveness. The amoeboid mode of invasion in these cell lines could be induced, e.g., through the addition of protease inhibitors, but this does not ensure that the cells are fully adapted for effective amoeboid invasiveness. To evaluate the plausibility of amoeboid invasion in native collagen gel, it would be much more suitable to use the metastatic cells, which use amoeboid invasion as their primary mode of invasiveness (e.g., A311 sarcoma cells; [15]) and which are genetically adapted for effective amoeboid invasiveness. It is possible that these cells could exhibit effective amoeboid invasiveness not only in native collagen gels but also in tissue in vivo.

\section{Conclusions}

The amoeboid and mesenchymal types of invasiveness are two modes of migration that are mutually interchangeable. Mesenchymal-amoeboid and amoeboid-mesenchymal transitions involve rapid changes in a migratory mode that arise as a reply to the current specifics of the environment. The definition of the particular types of movement that cells use after a transition is problematic. In most of the reported transitions, the feature determining the transition from mesenchymal to amoeboid invasiveness is the spherical cell morphology in the $3 \mathrm{D}$ environment. So far, only limited number of studies describing molecular mechanisms inducing MAT/AMT has been published. In the $3 \mathrm{D}$ environment, the treatments affecting the MAT and AMT were reported only in specific cell lines; thus, the universality of the specific treatment has to be further confirmed in other cell types. On the molecular level, the transitions are generally associated with changes in P-MLC2 levels or the actin localization and related features as well as the increased RhoA activity or membrane blebbing. The issue is that it is not known whether all the transitions observed are "complete" or whether it is possible for a specific treatment to trigger "partial" transitions 


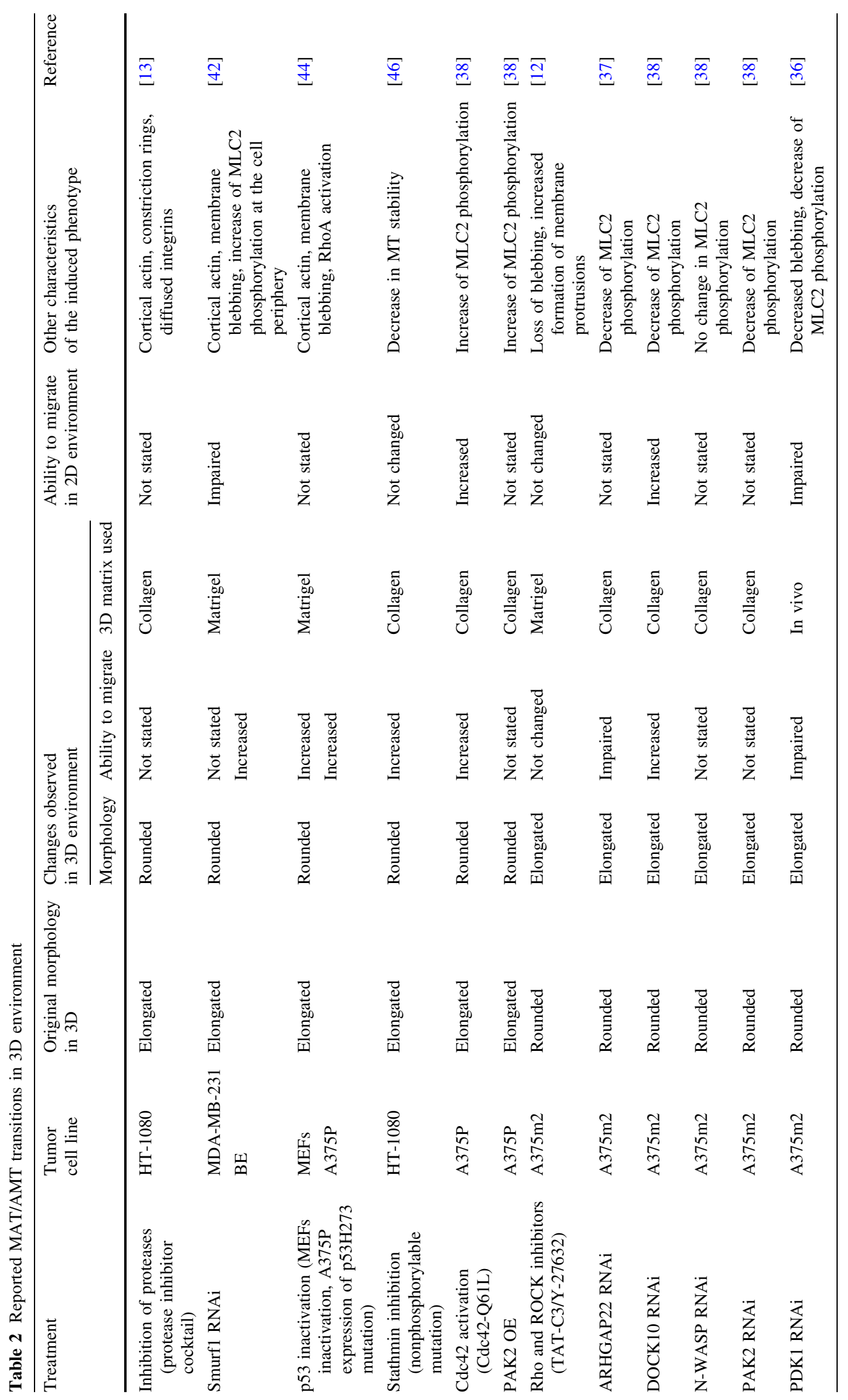


where the resultant phenotype is a mixture of the mesenchymal and amoeboid characteristics. Therefore, exact definitions for determining the transitions should be set.

Acknowledgment This work was supported by grants from the Czech Ministry of Education, Youth and Sport (Research Center grant LC06061 and LC07032) and (MSM0021620858). We thank our reviewer for useful comments.

Open Access This article is distributed under the terms of the Creative Commons Attribution Noncommercial License which permits any noncommercial use, distribution, and reproduction in any medium, provided the original author(s) and source are credited.

\section{References}

1. Kopfstein L, Christofori G (2006) Metastasis: cell-autonomous mechanisms versus contributions by the tumor microenvironment. Cell Mol Life Sci 63:449-468

2. Ridley AJ, Paterson HF, Johnston CL, Diekmann D, Hall A (1992) The small GTP-binding protein Rac regulates growth factor-induced membrane ruffling. Cell 70:401-410

3. Nobes CD, Hall A (1995) Rho, Rac, and Cdc42 GTPases regulate the assembly of multimolecular focal complexes associated with actin stress fibers, lamellipodia, and filopodia. Cell 81:53-62

4. Sheetz MP, Felsenfeld DP, Galbraith CG (1998) Cell migration: regulation of force on extracellular-matrix-integrin complexes. Trends Cell Biol 8:51-54

5. Brooks PC, Strömblad S, Sanders LC, von Schalscha TL, Aimes RT, Stetler-Stevenson WG, Quigley JP, Cheresh DA (1996) Localization of matrix metalloproteinase MMP-2 to the surface of invasive cells by interaction with integrin alpha $v$ beta 3 . Cell 85:683-693

6. Wei Y, Lukashev M, Simon DI, Bodary SC, Rosenberg S, Doyle MV, Chapman HA (1996) Regulation of integrin function by the urokinase receptor. Science 273:1551-1555

7. Friedl P, Zänker KS, Bröcker EB (1998) Cell migration strategies in 3-D extracellular matrix: differences in morphology, cell matrix interactions, and integrin function. Microsc Res Tech 43:369-378

8. Palecek SP, Loftus JC, Ginsberg MH, Lauffenburger DA, Horwitz AF (1997) Integrin-ligand binding properties govern cell migration speed through cell-substratum adhesiveness. Nature 385:537-540

9. Yumura S, Mori H, Fukui Y (1984) Localization of actin and myosin for the study of ameboid movement in Dictyostelium using improved immunofluorescence. J Cell Biol 99:894-899

10. Mandeville JT, Lawson MA, Maxfield FR (1997) Dynamic imaging of neutrophil migration in three dimensions: mechanical interactions between cells and matrix. J Leukoc Biol 61:188-200

11. Friedl P, Borgmann S, Bröcker EB (2001) Amoeboid leukocyte crawling through extracellular matrix: lessons from the Dictyostelium paradigm of cell movement. J Leukoc Biol 70:491-509

12. Sahai E, Marshall CJ (2003) Differing modes of tumour cell invasion have distinct requirements for Rho/ROCK signalling and extracellular proteolysis. Nat Cell Biol 5:711-719

13. Wolf $\mathrm{K}$, Mazo I, Leung $\mathrm{H}$, Engelke $\mathrm{K}$, von Andrian UH, Deryugina EI, Strongin AY, Bröcker EB, Friedl P (2003) Compensation mechanism in tumor cell migration: mesenchymalamoeboid transition after blocking of pericellular proteolysis. J Cell Biol 160:267-277

14. Wyckoff JB, Pinner SE, Gschmeissner S, Condeelis JS, Sahai E (2006) ROCK- and myosin-dependent matrix deformation enables protease-independent tumor-cell invasion in vivo. Curr Biol 16:1515-1523

15. Rösel D, Brábek J, Tolde O, Mierke CT, Zitterbart DP, Raupach C, Bicanová K, Kollmannsberger P, Panková D, Vesely P, Folk P, Fabry B (2008) Up-regulation of Rho/ROCK signaling in sarcoma cells drives invasion and increased generation of protrusive forces. Mol Cancer Res 6:1410-1420

16. Provenzano PP, Inman DR, Eliceiri KW, Trier SM, Keely PJ (2008) Contact guidance mediated three-dimensional cell migration is regulated by $\mathrm{Rho} / \mathrm{ROCK}$-dependent matrix reorganization. Biophys J 95:5374-5384

17. Keller H, Eggli P (1998) Protrusive activity, cytoplasmic compartmentalization, and restriction rings in locomoting blebbing Walker carcinosarcoma cells are related to detachment of cortical actin from the plasma membrane. Cell Motil Cytoskeleton 41:181-193

18. Friedl P, Noble PB, Shields ED, Zänker KS (1994) Locomotor phenotypes of unstimulated CD45RAhigh and CD45ROhigh $\mathrm{CD} 4+$ and $\mathrm{CD} 8+$ lymphocytes in three-dimensional collagen lattices. Immunology 82:617-624

19. Miki H, Suetsugu S, Takenawa T (1998) WAVE, a novel WASPfamily protein involved in actin reorganization induced by Rac. EMBO J 17:6932-6941

20. Rohatgi R, Ma L, Miki H, Lopez M, Kirchhausen T, Takenawa T, Kirschner MW (1999) The interaction between N-WASP and the Arp2/3 complex links Cdc42-dependent signals to actin assembly. Cell 97:221-231

21. Suetsugu $\mathrm{S}$, Miki $\mathrm{H}$, Yamaguchi $\mathrm{H}$, Obinata $\mathrm{T}$, Takenawa $\mathrm{T}$ (2001) Enhancement of branching efficiency by the actin filament-binding activity of N-WASP/WAVE2. J Cell Sci 114: 4533-4542

22. Yang L, Wang L, Zheng Y (2006) Gene targeting of Cdc42 and Cdc42GAP affirms the critical involvement of Cdc42 in filopodia induction, directed migration, and proliferation in primary mouse embryonic fibroblasts. Mol Biol Cell 17:4675-4685

23. Cau J, Hall A (2005) Cdc42 controls the polarity of the actin and microtubule cytoskeletons through two distinct signal transduction pathways. J Cell Sci 118:2579-2587

24. Calderwood DA, Zent R, Grant R, Rees DJ, Hynes RO, Ginsberg MH (1999) The Talin head domain binds to integrin beta subunit cytoplasmic tails and regulates integrin activation. J Biol Chem 274:28071-28074

25. Zaidel-Bar R, Cohen M, Addadi L, Geiger B (2004) Hierarchical assembly of cell-matrix adhesion complexes. Biochem Soc Trans 32:416-420

26. Kimura K, Ito M, Amano M, Chihara K, Fukata Y, Nakafuku M, Yamamori B, Feng J, Nakano T, Okawa K, Iwamatsu A, Kaibuchi K (1996) Regulation of myosin phosphatase by Rho and Rho-associated kinase (Rho-kinase). Science 273: 245-248

27. Amano M, Ito M, Kimura K, Fukata Y, Chihara K, Nakano T, Matsuura Y, Kaibuchi K (1996) Phosphorylation and activation of myosin by Rho-associated kinase (Rho-kinase). J Biol Chem 271:20246-20249

28. Mierke CT, Rösel D, Fabry B, Brábek J (2008) Contractile forces in tumor cell migration. Eur J Cell Biol 87:669-676

29. Wilkinson S, Paterson HF, Marshall CJ (2005) Cdc42-MRCK and Rho-ROCK signalling cooperate in myosin phosphorylation and cell invasion. Nat Cell Biol 7:255-261

30. Leung T, Chen XQ, Tan I, Manser E, Lim L (1998) Myotonic dystrophy kinase-related Cdc42-binding kinase acts as a $\mathrm{Cdc} 42$ effector in promoting cytoskeletal reorganization. Mol Cell Biol 18:130-140

31. Yanai M, Kenyon CM, Butler JP, Macklem PT, Kelly SM (1996) Intracellular pressure is a motive force for cell motion in Amoeba proteus. Cell Motil Cytoskeleton 33:22-29 
32. Niggli V, Rossy J (2008) Ezrin/radixin/moesin: versatile controllers of signaling molecules and of the cortical cytoskeleton. Int J Biochem Cell Biol 40:344-349

33. Charras GT, Hu CK, Coughlin M, Mitchison TJ (2006) Reassembly of contractile actin cortex in cell blebs. J Cell Biol 175:477-490

34. Hegerfeldt Y, Tusch M, Bröcker EB, Friedl P (2002) Collective cell movement in primary melanoma explants: plasticity of cellcell interaction, beta1-integrin function, and migration strategies. Cancer Res 62:2125-21230

35. Carragher NO, Walker SM, Scott Carragher LA, Harris F, Sawyer TK, Brunton VG, Ozanne BW, Frame MC (2006) Calpain 2 and $\mathrm{Src}$ dependence distinguishes mesenchymal and amoeboid modes of tumour cell invasion: a link to integrin function. Oncogene 25:5726-5740

36. Pinner S, Sahai E (2008) PDK1 regulates cancer cell motility by antagonising inhibition of ROCK1 by RhoE. Nat Cell Biol 10:127-137

37. Sanz-Moreno V, Gadea G, Ahn J, Paterson H, Marra P, Pinner S, Sahai E, Marshall CJ (2008) Rac activation and inactivation control plasticity of tumor cell movement. Cell 135:510-523

38. Gadea G, Sanz-Moreno V, Self A, Godi A, Marshall CJ (2008) DOCK10-mediated Cdc42 activation is necessary for amoeboid invasion of melanoma cells. Curr Biol 18:1456-1465

39. Bosco EE, Mulloy JC, Zheng Y (2009) Rac1 GTPase: a "Rac" of all trades. Cell Mol Life Sci 66:370-374

40. Guo F, Debidda M, Yang L, Williams DA, Zheng Y (2006) Genetic deletion of Rac1 GTPase reveals its critical role in actin stress fiber formation and focal adhesion complex assembly. J Biol Chem 281:18652-18659
41. Wang HR, Ogunjimi AA, Zhang Y, Ozdamar B, Bose R, Wrana JL (2006) Degradation of RhoA by Smurf1 ubiquitin ligase. Methods Enzymol 406:437-447

42. Sahai E, Garcia-Medina R, Pouysségur J, Vial E (2007) Smurf1 regulates tumor cell plasticity and motility through degradation of RhoA leading to localized inhibition of contractility. J Cell Biol 176:35-42

43. Gadea G, Lapasset L, Gauthier-Rouvière C, Roux P (2002) Regulation of Cdc42-mediated morphological effects: a novel function for p53. EMBO J 21:2373-2382

44. Gadea G, de Toledo M, Anguille C, Roux P (2007) Loss of p53 promotes RhoA-ROCK-dependent cell migration and invasion in 3D matrices. J Cell Biol 178:23-30

45. Curmi PA, Gavet O, Charbaut E, Ozon S, Lachkar-Colmerauer S, Manceau V, Siavoshian S, Maucuer A, Sobel A (1999) Stathmin and its phosphoprotein family: general properties, biochemical and functional interaction with tubulin. Cell Struct Funct 24: 345-357

46. Belletti B, Nicoloso MS, Schiappacassi M, Berton S, Lovat F, Wolf K, Canzonieri V, D'Andrea S, Zucchetto A, Friedl P, Colombatti A, Baldassarre G (2008) Stathmin activity influences sarcoma cell shape, motility, and metastatic potential. Mol Biol Cell 19:2003-2013

47. Niggli V (2003) Microtubule-disruption-induced and chemotactic-peptide-induced migration of human neutrophils: implications for differential sets of signalling pathways. J Cell Sci 116: 813-822

48. Sabeh F, Shimizu-Hirota R, Weiss SJ (2009) Protease-dependent versus -independent cancer cell invasion programs: three-dimensional amoeboid movement revisited. J Cell Biol 185:11-19 